\title{
ON STRONG AUTOMORPHISMS OF DIRECT PRODUCTS OF WITT RINGS (II)
}

\author{
Marcin Ryszard Stepien ${ }^{1}$, Lidia Stępien ${ }^{2}$ \\ ${ }^{I}$ Chair of Mathematics, Kielce University of Technology \\ Kielce, Poland \\ ${ }^{2}$ Institute of Mathematics and Computer Science, Jan Dtugosz University \\ Czestochowa, Poland \\ ${ }^{I}$ mstepien@tu.kielce.pl, ${ }^{2}$ l.stepien@ajd.czest.pl
}

\begin{abstract}
In the paper strong automorphisms of finitely generated Witt rings are considered. Every finitely generated Witt ring can be expressed in terms of $\mathbb{Z} / 2 \mathbb{Z}$ and basic indecomposable Witt rings using the operations of group ring formation and direct product. Groups of strong automorphisms of basic indecomposables and their direct products and the description of all strong automorphisms of any group Witt ring are known. In this paper the strong automorphisms of direct products of group Witt rings are considered. Presented are two wide classes of Witt rings where the group of strong automorphisms is isomorphic to the direct product of groups of strong automorphisms of Witt rings which are factors in the direct product.
\end{abstract}

Keywords: Witt rings, strong automorphisms, group rings

\section{Introduction}

We consider abstract Witt rings in terminology of M. Marshall [1]. We shall describe the groups of strong automorphisms of some direct products of group Witt rings. The results presented here are continuation of what is included in [2]. Therefore we omit some definitions (in particular the definition of abstract Witt ring) and facts and limit preliminaries to the minimum necessary in the paper. For further information the reader can go to [1] or [2].

Let the pair $W=(R, G)$ be a Witt ring, which is finitely generated by the group $G$. As we know there is one-to-one correspondence between Witt rings and quaternionic structures $([1$, Theorem 4.5]). The correspondence extends to their automorphisms and the group of strong automorphisms of Witt ring $W$ is isomorphic to the group of automorphisms of the quaternionic structure $(G, Q, q)$ associated to $W$ (compare [3]). Since we shall use the properties of quaternionic structures, we recall the definition.

Definition 1.1. Let $G$ be a group of exponent 2, i.e. $a^{2}=1$ for all $a \in G$ with distinguished element $-1 \in G$ and let us denote $-a=-1 \cdot a$. Let $Q$ be the set with 
distinguished element $\theta$ and let $q: G \times G \rightarrow Q$ be a surjective map. The triplet $(G, Q, q)$ is called a quaternionic structure if for every $a, b, c, d \in G$ the map $q$ fulfills:

$Q_{1}: q(a, b)=q(b, a)$

$Q_{2}: q(a,-a)=\theta$

$Q_{3}: q(a, b)=q(a, c) \Rightarrow q(a, b c)=\theta$

$Q_{4}$ : If $q(a, b)=q(c, d)$, then there exists such $x \in G$ that $q(a, b)=q(a, x)$ and $q(c, d)=q(c, x)$.

Let $(G, Q, q)$ be a quaternionic structure. A (quadratic) form of dimension $n \geq 1$ over $G$ is $n$-tuple $f=\left(a_{1}, \ldots, a_{n}\right)$, where $a_{1}, \ldots, a_{n} \in G$. Two forms of dimension $n$ are called equivalent if:
(1) $n=1$,
$(a) \cong(b) \Leftrightarrow a=b$
(2) $n=2$,
$(a, b) \cong(c, d) \Leftrightarrow a b=c d$ and $q(a, b)=q(c, d)$
(3) $n>2$,
$\left(a_{1}, \ldots, a_{n}\right) \cong\left(b_{1}, \ldots, b_{n}\right) \Leftrightarrow \exists a, b, c_{3}, \ldots, c_{n} \in G$ such that
$\left(a_{2}, \ldots, a_{n}\right) \cong\left(a, c_{3}, \ldots, c_{n}\right), \quad\left(a_{1}, a\right) \cong\left(b_{1}, b\right)$ and $\left(b_{2}, \ldots, b_{n}\right) \cong$ $\cong\left(b, c_{3}, \ldots, c_{n}\right)$.

The form $\left(1, a_{1}\right) \otimes \cdots \otimes\left(1, a_{n}\right)$, where $a_{1}, \ldots, a_{n} \in G, n>0$ is named $n$-fold Pfister form. We say that form frepresents element $a \in G$ if there exist $a_{2}, \ldots, a_{n} \in$ $\in G$, such that $f \cong\left(a, a_{2}, \ldots a_{n}\right)$. The set of all elements represented by form $f$ (value set of the form $f$ ) is denoted by $D(f)$.

Let $W=(R, G)$ be a Witt ring and let $(G, Q, q)$ be corresponding quaternionic structure. By strong automorphism of $W$ we understand any ring automorphism $\varphi \in \operatorname{Aut}(R)$ such that $\varphi(G)=G$. By [3, Theorem 2.1] there exists a corresponding automorphism of quaternionic structure $\sigma$, i.e. a group automorphism $\sigma \in \operatorname{Aut}(G)$ such that $\sigma(-1)=-1$ and $q(a, b)=\theta \Leftrightarrow q(\sigma(a), \sigma(b))=\theta$ for all $a, b \in G$.

We say that a Witt ring $W=(R, G)$ is indecomposable if firstly $W \neq \mathbb{Z} / 2 \mathbb{Z}$, and secondly, if $(R, G) \cong\left(R_{1}, G_{1}\right) \sqcap\left(R_{2}, G_{2}\right)$ implies either $R_{1} \cong \mathbb{Z} / 2 \mathbb{Z}$ or $R_{2} \cong \mathbb{Z} / 2 \mathbb{Z}$ ([1]). An element $a \in G$ is rigid if $b \in D(1, a) \Rightarrow b=1$ or $b=a$ or the value set of the form $(1, a)$ is just $\{1, a\}$. If $G \neq\{1,-1\}$ we will say that $a \in G$ is basic if either $a$ or $-a$ is not rigid. In case $G=\{1,-1\}$ we consider -1 and 1 both to be basic. The elements in the group $G$, which are not basic we will call birigid. A Witt ring $W=(R, G)$ is said to be basic Witt ring if all elements of $G$ are basic. A Witt ring, which is both basic and indecomposable is, called basic indecomposable Witt ring [compare [1]).

M. Marshall proved that any finitely generated Witt ring can be expressed in terms of $\mathbb{Z} / 2 \mathbb{Z}$ and basic indecomposable Witt rings using the operations of group ring formation and direct product ([1, Theorem 5.23]). M. Marshall in [1] presented the all known basic indecomposables as $\mathbb{Z} / 4 \mathbb{Z}, \mathbb{Z}$, and Witt rings of local type. Therefore, if we want to find strong automorphisms of finitely generated Witt rings we should know how to describe the groups of automorphisms of Witt rings of local type, group Witt rings and their direct products. We know the description of strong automorphisms of Witt rings of local type ([4]), their direct products ([5]) 
and group Witt rings ([6]). In this paper we show that in many cases the group of strong automorphisms of direct product of group Witt rings can be precisely described.

\section{Automorphisms of direct products of group Witt rings}

Let $W=(R, G)$ be finite direct product of Witt rings $W_{1}=\left(R_{1}, G_{1}\right), \ldots, W_{n}=$ $=\left(R_{n}, G_{n}\right)$ which are not pairwise (strongly) isomorphic. Let $(G, Q, q)$ be the quaternionic structure associated to $W$ and assume that $(G, Q, q)=$ $=\prod_{i=1}^{n}\left(G_{i}, Q_{i}, q_{i}\right)$. Denote the subgroup $\{1\} \times \cdots \times\{1\} \times G_{k} \times\{1\} \times \cdots \times\{1\}$ of the group $G=G_{1} \times \cdots \times G_{n}$ by $G_{k}^{\prime}$, where $1 \leq k \leq n$. There was shown in [2] that the necessary condition for $\operatorname{Aut}(G, Q, q) \cong \prod_{i=1}^{n} \operatorname{Aut}\left(G_{i}, Q_{i}, q_{i}\right)$ (and consequently $\left.\operatorname{Aut}(W) \cong \prod_{i=1}^{n} \operatorname{Aut}\left(W_{i}\right)\right)$ is that any automorphism of the group $G$ preserves the factors of the product i.e. if for all $k \in\{1, \ldots, n\}$ there exists $j \in\{1, \ldots, n\}$ such that $\sigma\left(G_{k}^{\prime}\right)=G_{j}^{\prime}$.

Below we present two broad classes of direct products of group Witt rings where the condition described above is fulfilled.

Proposition 2.1. Let $\Delta_{n}$ be a group of exponent 2 with cardinality $2^{n}, n \in \mathbb{N}$. Let $\left(G_{1}, Q_{1}, q_{1}\right)$ be a quaternionic structure associated to the Witt ring $W_{1}=\left(S\left[\Delta_{1}\right], G_{1}\right)$, where $S$ is a basic Witt ring and let $\left(G_{2}, Q_{2}, q_{2}\right)$ be the quaternionic structure associated to Witt ring $W_{2} \cong W\left(\mathbb{Q}_{5}\right)\left[\Delta_{n}\right]$. If $\left|G_{1}\right|=2^{m}$, then for $n \geq m-1$ and for $\sigma \in \operatorname{Aut}(G, Q, q)=\operatorname{Aut}\left(G_{1} \times G_{2}, Q_{1} \times Q_{2}, q_{1} \times q_{2}\right)$ holds:

(i) $\sigma\left(G_{1} \times\left\{1_{2}\right\}\right)=G_{1} \times\left\{1_{2}\right\}$ and

(ii) $\sigma\left(\left\{1_{2}\right\} \times G_{2}\right)=\left\{1_{2}\right\} \times G_{2}$.

Proof. Let us calculate the possible cardinalities of value sets of binary Pfister forms $\left(1_{i}, b\right)$, where elements $b$ are from $G_{i}, i=1,2$.

At least a half of elements of the group $G_{1}$ are birigid, hence we have $\left|D_{1}\left(1_{1}, b\right)\right|=2$ if $b$ is birigid. The remaining elements of $G_{1}$ fulfill $\left|D_{1}\left(1_{1}, b^{\prime}\right)\right| \leq$ $\leq 2^{m}$ (in particular $\left|D_{1}\left(1_{1},-1_{1}\right)\right|=2^{m}$ ).

In $G_{2}$ we have $1_{2}=-1_{2}$, then $\left|D_{2}\left(1_{2},-1_{2}\right)\right|=2^{n+2}$ and $\left|D_{2}\left(1_{2}, b\right)\right|=2$ if $b \neq 1_{2}$ (since the group $G_{\mathbb{Q}_{5}}$ has 4 elements).

Notice first that $|D(\mathbf{1},-\mathbf{1})|=\left|D\left(\left[1_{1}, 1_{2}\right],\left[-1_{1}, 1_{2}\right]\right)\right|=\left|D_{1}\left(1_{1},-1_{1}\right)\right|$. $\left|D_{2}\left(1_{2}, 1_{2}\right)\right|=2^{m} \cdot 2^{n+2}$.

Now let us consider an element $\boldsymbol{c}$ such that $\left[1_{1}, 1_{2}\right] \neq \boldsymbol{c}=\left[c_{1}, c_{2}\right] \in G_{1} \times G_{2}$. We have $|D(\mathbf{1}, \boldsymbol{c})|=\left|D\left(\left[1_{1}, 1_{2}\right],\left[c_{1}, c_{2}\right]\right)\right|=\left|D_{1}\left(1_{1}, c_{1}\right) \times D_{2}\left(1_{2}, c_{2}\right)\right|=$ $=\left|D_{1}\left(1_{1}, c_{1}\right)\right| \cdot\left|D_{2}\left(1_{2}, c_{2}\right)\right|$. Then

$$
\begin{gathered}
|D(\mathbf{1}, \boldsymbol{c})| \leq 2^{m} \cdot 2=2^{m+1} \text { if } c_{2} \neq 1_{2} \\
|D(\mathbf{1}, \boldsymbol{c})| \geq 2 \cdot 2^{n+2}=2^{n+3} \geq 2^{m+2} \text { if } c_{2}=1_{2}
\end{gathered}
$$


Let $\boldsymbol{a}=\left[x, 1_{2}\right] \in G_{1} \times G_{2}$. Then by $(2.2)$ we get $|D(\mathbf{1}, \boldsymbol{a})| \geq 2^{m+2}$. Since $\sigma$ is an automorphism of quaternionic structure $(G, Q, q)$, then we also have $|D(\mathbf{1}, \sigma(\boldsymbol{a}))| \geq 2^{m+2}$.

Suppose that $\sigma(\boldsymbol{a})=\sigma\left(\left[x, 1_{2}\right]\right)=\left[x^{\prime}, y^{\prime}\right] \in G_{1} \times G_{2}$ and $y^{\prime} \neq 1_{2}$. Then $\left|D_{2}\left(1_{2}, y^{\prime}\right)\right|=2$ and from $y^{\prime} \neq 1_{2}$ it follows that $|D(\mathbf{1}, \sigma(\boldsymbol{a}))|=\left|D\left(\mathbf{1},\left[x^{\prime}, y^{\prime}\right]\right)\right|=$ $=\left|D_{1}\left(1_{1}, x^{\prime}\right)\right| \cdot\left|D_{2}\left(1_{2}, y^{\prime}\right)\right| \leq 2^{m} \cdot 2=2^{m+1}$ - a contradiction. Therefore we have $\sigma(\boldsymbol{a})=\sigma\left(\left[x, 1_{2}\right]\right)=\left[x^{\prime}, 1_{2}\right]$ for some $x^{\prime} \in G_{1}$ and since $\boldsymbol{a}=\left[x, 1_{2}\right]$ is any element in $G_{1} \times G_{2}$ which means that the condition (i) is fulfilled.

Let us now take an element $\boldsymbol{b}=\left[1_{1}, y\right]$ such that $y \neq 1_{2}$. Then $\left|D_{2}\left(1_{2}, y\right)\right|=2$ and by $(2.1)$ it follows that $|D(\mathbf{1}, \boldsymbol{b})| \leq 2^{m+1}$. Consider the opposite element $-\boldsymbol{b}=\left[-1_{1},-y\right]$, where $-y \neq 1_{2}$. Then $|D(\mathbf{1},-\boldsymbol{b})|=\left|D\left(\left[1_{1}, 1_{2}\right],\left[-1_{1},-y\right]\right)\right|=$ $=\left|D_{1}\left(1_{1},-1_{1}\right)\right| \cdot\left|D_{2}\left(1_{2},-y\right)\right|=2^{m} \cdot 2$, hence also $|D(\mathbf{1}, \sigma(-\boldsymbol{b}))|=2^{m+1}$.

Assume that $\sigma(\boldsymbol{b})=\left[x^{\prime \prime}, y^{\prime \prime}\right]$. By $|D(\mathbf{1}, \sigma(-\boldsymbol{b}))|=2^{m+1}$ and by $(2.1)$ it follows that $y^{\prime \prime} \neq 1_{2}$ (thus we have $\left|D_{2}\left(1_{2}, y^{\prime \prime}\right)\right|=2$ ). On the other hand $\sigma(-\boldsymbol{b})=$ $=\left[-x^{\prime \prime},-y^{\prime \prime}\right]$ and $y^{\prime \prime} \neq 1_{2}$, hence also $-y^{\prime \prime} \neq 1_{2}$, thus $\left|D_{2}\left(1_{2},-y^{\prime \prime}\right)\right|=2$. If $x^{\prime \prime} \neq 1_{1}$, then $-x^{\prime \prime} \neq-1$ and then $|D(\mathbf{1}, \sigma(-\boldsymbol{b}))|=\left|D\left(\left[1_{1}, 1_{2}\right],\left[-x^{\prime \prime},-y^{\prime \prime}\right]\right)\right|=$ $=\left|D_{1}\left(1_{1},-x^{\prime \prime}\right)\right| \cdot\left|D_{2}\left(1_{2},-y^{\prime \prime}\right)\right|<2^{m} \cdot 2=2^{m+1}-$ a contradiction. Therefore $x^{\prime \prime}=1_{1}$, hence $\sigma(\boldsymbol{b})=\sigma([1, y])=\left[1, y^{\prime \prime}\right]$, which means that the condition (ii) is fulfilled.

The following proposition concerns another class of direct products of group Witt rings and is similar to Proposition 2.1.

Proposition 2.2. Let $\Delta_{n}$ be a group of exponent 2 with cardinality $2^{n}, n \in \mathbb{N}$. Let $\left(G_{1}, Q_{1}, q_{1}\right)$ be a quaternionic structure associated to the Witt ring $W_{1}=\left(W\left(\mathbb{Q}_{3}\right) \sqcap W\left(\mathbb{Q}_{5}\right)\right)\left[\Delta_{1}\right]$. and let $\left(G_{2}, Q_{2}, q_{2}\right)$ be the quaternionic structure associated to Witt ring $W_{2} \cong W\left(\mathbb{Q}_{5}\right)\left[\Delta_{n}\right]$.

Then for $\sigma \in \operatorname{Aut}(G, Q, q)=\operatorname{Aut}\left(G_{1} \times G_{2}, Q_{1} \times Q_{2}, q_{1} \times q_{2}\right)$ holds:

(i) $\sigma\left(G_{1} \times\left\{1_{2}\right\}\right)=G_{1} \times\left\{1_{2}\right\}$ and

(ii) $\sigma\left(\left\{1_{2}\right\} \times G_{2}\right)=\left\{1_{2}\right\} \times G_{2}$.

Proof. Let us use notation $W_{1}=\left(R_{1}, G_{1}\right)$ and $W_{2}=\left(R_{2}, G_{2}\right)$. Let us first describe the groups $G_{1}$ and $G_{2}$ and value sets of 1-fold Pfister forms in quaternionic structures associated to $W_{1}$ and $W_{2}$. We shall use this information in our proof.

The ring $W_{1}$ is a group Witt ring of the group $\Delta_{1}=\{1, x\}$ with coefficients in Witt ring $W\left(\mathbb{Q}_{3}\right) \sqcap W\left(\mathbb{Q}_{5}\right)$ being a direct product of Witt rings of local type. As we know the groups $G_{\mathbb{Q}_{3}}$ and $G_{\mathbb{Q}_{5}}$ can be represented in the form: $G_{\mathbb{Q}_{3}}=$ $=\{1,-1, p,-p\}$ for some $p \in \mathbb{Q}_{3}, p \neq \pm 1_{\mathbb{Q}_{3}}$ and $G_{\mathbb{Q}_{5}}=\{1, p, u, u p\}$ for some $u, p \in \mathbb{Q}_{5}, u, p \neq 1_{\mathbb{Q}_{5}}=-1_{\mathbb{Q}_{5}}$ (compare [7, Theorem 2.2, p. 152] or [8, Corollary on p. 18]). Using a definition of elements represented by forms in the direct products of Witt rings and in group Witt rings we can describe value sets of 1-fold Pfister forms in $W_{1}$ in the following way. 
1) $\left|D_{1}\left(1_{1},-1_{1}\right)\right|=32$.

2) There are 16 birigid elements, i.e. such that $\left|D_{1}\left(1_{1}, d\right)\right|=2$, where $\pm 1_{1} \neq d \in$ $\in G_{1}$ is of the form $d=\left[d^{\prime}, x\right], x \in \Delta_{1}, d^{\prime} \in G_{\mathbb{Q}_{3}} \times G_{\mathbb{Q}_{3}}, x \in \Delta_{1}$ (then $\left.D_{1}\left(1_{1}, d\right)=\left\{1_{1}, d\right\}\right)$.

3) There are 9 elements of the form $\pm 1_{1} \neq d \neq\left[d^{\prime}, 1\right] \in\left(G_{\mathbb{Q}_{3}} \times G_{\mathbb{Q}_{5}}\right) \times \Delta_{1}$, such that $\left|D_{1}\left(1_{1}, d\right)\right|=4$.

4) There are 6 elements of the form $-1_{1} \neq d=[b, 1] \in\left(G_{\mathbb{Q}_{3}} \times G_{\mathbb{Q}_{5}}\right) \times \Delta_{1}$, such that $\left|D_{1}\left(1_{1}, d\right)\right|=8$ (in particular $\left|D_{1}\left(1_{1}, 1_{1}\right)\right|=8$ ).

The ring $W_{2}$ is the group Witt ring of a group $\Delta_{n}$ of exponent 2 (for $n \geq 1$ ) with coefficients in Witt ring of local type $W\left(\mathbb{Q}_{5}\right)$. We have $G_{2}=G_{\mathbb{Q}_{5}} \times \Delta_{n}$, hence $\left|G_{2}\right|=2^{2} \cdot 2^{n}=2^{n+2}$. Therefore we can describe value sets of 1 -fold Pfister forms as follows.

1) $D_{2}\left(1_{2}, 1_{2}\right)=D_{2}\left(1_{2},-1_{2}\right)=G_{2}$.

2) There are 3 elements of the form $1_{2} \neq d=\left[d^{\prime}, 1\right] \in G_{\mathbb{Q}_{5}} \times \Delta_{n}$ such that $\left|D_{2}\left(1_{2}, d\right)\right|=2$.

3) There are $2^{n+2}-4$ elements of the form $d=\left[d^{\prime}, d^{\prime \prime}\right] \in G_{\mathbb{Q}_{5}} \times \Delta_{n}, d^{\prime} \neq 1_{2}$ in $G_{\mathbb{Q}_{5}}, d^{\prime \prime} \neq 1$ in $\Delta_{n}$ such that $\left|D_{2}\left(1_{2}, d\right)\right|=2$.

Notice that in the case $n=0$ (or for the Witt ring $W\left(\mathbb{Q}_{5}\right)$ ) such elements do not exist!

Now we are going to the main part of our proof.

Notice first that $1_{2}=-1_{2} \in G_{2}$, hence $\left[1_{1},-1_{2}\right]=\left[1_{1}, 1_{2}\right]=\mathbf{1}$ and $\left[-1_{1}, 1_{2}\right]=$ $=\left[-1_{1},-1_{2}\right]=-\mathbf{1} \in G_{1} \times G_{2}$, thus for any automorphism $\sigma \in \operatorname{Aut}(G, Q, q)$ we get $\sigma\left(\left[1_{1},-1_{2}\right]\right)=\left[1_{1},-1_{2}\right]$ and $\sigma\left(\left[-1_{1}, 1_{2}\right]\right)=\left[-1_{1}, 1_{2}\right]$.

Let us consider an element $\boldsymbol{a}=\left[1_{1}, y\right] \in G_{1} \times G_{2}$ such that $y \neq 1_{2}$. Then $|D(\mathbf{1}, \boldsymbol{a})|=\left|D_{1}\left(1_{1}, 1_{1}\right) \times D_{2}\left(1_{2}, y\right)\right|=8 \cdot 2=16$ and for the opposite element $-a=\left[-1_{1},-y\right]$ we have $|D(\mathbf{1},-\boldsymbol{a})|=\left|D_{1}\left(1_{1},-1_{1}\right) \times D_{2}\left(1_{2},-y\right)\right|=32 \cdot 2=$ $=64$. Since $\sigma$ is an automorphism of quaternionic structure and it preserves value sets of forms, then we get also $|D(\mathbf{1}, \sigma(\boldsymbol{a}))|=16$ and $|D(\mathbf{1}, \sigma(-\boldsymbol{a}))|=64$. Assume that $\sigma(\boldsymbol{a})=\sigma\left(\left[1_{1}, y\right]\right)=\left[x^{\prime}, y^{\prime}\right] \in G_{1} \times G_{2}$. We have $16=|D(1, \sigma(\boldsymbol{a}))|=$ $=\left|D_{1}\left(1_{1}, x^{\prime}\right)\right| \cdot\left|D_{2}\left(1_{2}, y^{\prime}\right)\right|$. As we know there is impossible $\left|D_{1}\left(1_{1}, x^{\prime}\right)\right|=1$ and $\left|D_{2}\left(1_{2}, y^{\prime}\right)\right|=1$ in $G_{1} \times G_{2}$, therefore either of the three cases occur:

(1) $\left|D_{1}\left(1_{1}, x^{\prime}\right)\right|=2$ and $\left|D_{2}\left(1_{2}, y^{\prime}\right)\right|=8$ or

(2) $\left|D_{1}\left(1_{1}, x^{\prime}\right)\right|=4$ and $\left|D_{2}\left(1_{2}, y^{\prime}\right)\right|=4$ or

(3) $\left|D_{1}\left(1_{1}, x^{\prime}\right)\right|=8$ and $\left|D_{2}\left(1_{2}, y^{\prime}\right)\right|=2$.

We shall show, that the cases (1) and (2) are impossible and only case (3) holds.

Let us consider all above cases one after the other.

Ad (1). By the second condition $\left|D_{2}\left(1_{2}, y^{\prime}\right)\right|=8$ and by properties of the group $G_{2}$ it follows that $y^{\prime}=-1_{2}=1_{2} \in G_{2}$, which means that $y^{\prime}=-y^{\prime}=1_{2}$ and $\left|G_{2}\right|=8$ (or $n=1$ ). Therefore we have $\sigma(\boldsymbol{a})=\left[x^{\prime}, 1_{2}\right]$ for some $x^{\prime} \in G_{1}$, thus $\sigma(-\boldsymbol{a})=\left[-x^{\prime}, 1_{2}\right] . \quad$ Since $\quad 64=|D(\mathbf{1}, \sigma(-\boldsymbol{a}))|=\left|D\left(\left[1_{1}, 1_{2}\right],\left[-x^{\prime}, 1_{2}\right]\right)\right|=$ 
$=\left|D_{1}\left(1_{1},-x^{\prime}\right)\right| \cdot\left|D_{2}\left(1_{2}, 1_{2}\right)\right|$ and $\left|D_{2}\left(1_{2}, 1_{2}\right)\right|=8$, then $\left|D_{1}\left(1_{1},-x^{\prime}\right)\right|=8$. But from properties of $G_{1}$ we know that for any $x^{\prime} \in G_{1}$ such that $\left|D_{1}\left(1_{1},-x^{\prime}\right)\right|=8$ we have $\left|D_{1}\left(1_{1}, x^{\prime}\right)\right| \neq 2$ - a contradiction to (1).

Ad (2). By similar argumentation as in the previous case we get $\left|D_{2}\left(1_{2}, y^{\prime}\right)\right|=4$ implies $y^{\prime}=-y^{\prime}=1_{2} \in G_{2}$ and $\left|G_{2}\right|=8$ and $n=0$. Therefore we have $64=|D(\mathbf{1}, \sigma(-\boldsymbol{a}))|=\left|D\left(\left[1_{1}, 1_{2}\right],\left[-x^{\prime}, 1_{2}\right]\right)\right|=\left|D_{1}\left(1_{1},-x^{\prime}\right)\right| \cdot\left|D_{2}\left(1_{2}, 1_{2}\right)\right|$ and $\left|D_{2}\left(1_{2}, 1_{2}\right)\right|=4$, then $\left|D_{1}\left(1_{1},-x^{\prime}\right)\right|=16-$ a contradiction, because for any $d \in G_{1}$ we have $\left|D_{1}\left(1_{1}, d\right)\right| \neq 16$.

Therefore we have shown that the case (3) holds. Assuming that $\sigma\left(\left[1_{1}, y\right]\right)=$ $=\left[x^{\prime}, y^{\prime}\right]$ for some $x^{\prime} \in G_{1}, y^{\prime} \in G_{2}$ we shall show that $x^{\prime}=1_{1}$.

We have $\sigma(-\boldsymbol{a})=\left[-x^{\prime},-y^{\prime}\right]$ and $|D(\mathbf{1}, \sigma(-\boldsymbol{a}))|=64$. If $y^{\prime}=1_{2}$, then by condition (3) it follows that $\left|D_{2}\left(1_{2}, y^{\prime}\right)\right|=\left|D_{2}\left(1_{2}, 1_{2}\right)\right|=2$, hence $\left|G_{2}\right|=2$, a contradiction (since $\left|G_{2}\right| \geq 4$ ). Therefore $y^{\prime} \neq 1_{2}$ in $G_{2}$. Then $-y^{\prime} \neq 1_{2}$ and by properties of the group $G_{2}$ it follows that $\left|D_{2}\left(1_{2},-y^{\prime}\right)\right|=2$. Since $64=|D(\mathbf{1}, \sigma(-\boldsymbol{a}))|=\left|D_{1}\left(1_{1},-x^{\prime}\right)\right| \cdot\left|D_{2}\left(1_{2},-y^{\prime}\right)\right|, \quad$ then $\left|D_{1}\left(1_{1},-x^{\prime}\right)\right|=32$ and $-x^{\prime}=-1_{1} \in G_{1}$ and consequently $x^{\prime}=1_{1}$. It means that for every $y \in G_{2}$ we have $\sigma(\boldsymbol{a})=\sigma\left(\left[1_{1}, y\right]\right)=\left[1_{1}, y^{\prime}\right] \in G_{1} \times G_{2}$, which finishes the proof (ii).

Let $1_{2} \neq d, e \in G_{2}$ and $d \neq e$. Then by (ii) we can write $\sigma\left(\left[1_{1}, d\right]\right)=\left[1_{1}, d^{\prime}\right]$ and $\sigma\left(\left[1_{1}, e\right]\right)=\left[1_{1}, e^{\prime}\right]$ for some $d^{\prime}, e^{\prime} \in G_{2}$. In order to prove (i) we shall calculate $D\left(\left[1_{1}, 1_{2}\right],\left[-1_{1}, d\right]\right) \cap D\left(\left[1_{1}, 1_{2}\right],\left[-1_{1}, d^{\prime}\right]\right)$.

Let us first notice that

$D\left(\left[1_{1}, 1_{2}\right],\left[-1_{1}, d\right]\right) \cap D\left(\left[1_{1}, 1_{2}\right],\left[-1_{1}, e\right]\right)=$

$=\left(D_{1}\left(1_{1},-1_{1}\right) \times D_{2}\left(1_{2}, d\right)\right) \cap\left(D_{1}\left(1_{1},-1_{1}\right) \times D_{2}\left(1_{2}, e\right)\right)=$

$=\left(D_{1}\left(1_{1},-1_{1}\right) \cap D_{1}\left(1_{1},-1_{1}\right)\right) \times\left(D_{2}\left(1_{2}, d\right) \cap D_{2}\left(1_{2}, e\right)\right)=G_{1} \times\left\{1_{2}\right\}$.

Therefore for any $\sigma \in \operatorname{Aut}(G, Q, q)$ we get

$\sigma\left(G_{1} \times\left\{1_{2}\right\}\right)=$

$=\sigma\left(D\left(\left[1_{1}, 1_{2}\right],\left[\left[-1_{1}, d\right]\right) \cap D\left(\left[1_{1}, 1_{2}\right],\left[-1_{1}, e\right]\right)\right)=\right.$

$\left.=D\left(\left[1_{1}, 1_{2}\right]\right), \sigma\left(\left[-1_{1}, d\right]\right)\right) \cap D\left(\left[1_{1}, 1_{2}\right], \sigma\left(\left[-1_{1}, e\right]\right)\right)=$

$=D\left(\left[1_{1}, 1_{2}\right],\left[-1_{1}, d^{\prime}\right]\right) \cap D\left(\left[1_{1}, 1_{2}\right],\left[-1_{1}, e^{\prime}\right]\right)=G_{1} \times\left\{1_{2}\right\}$

which finishes the proof of (i).

Propositions 2.1 and 2.2 show that a lot of examples of direct products of group Witt rings which preserves their factors exists. In consequence we can calculate their groups of strong automorphisms as the direct product groups of automorphisms of factors of direct product. Unfortunately not every direct product of group Witt rings preserves the factors of direct product as it shows the following example.

Example 2.3. Let us consider a Witt ring $W=\mathbb{Z} / 2 \mathbb{Z}\left[\Delta_{1}\right] \sqcap \mathbb{Z} / 2 \mathbb{Z}\left[\Delta_{2}\right]$. As we know, the only strong automorphism of $\mathbb{Z} / 2 \mathbb{Z}\left[\Delta_{1}\right]$ is identity (compare $[3, \S 3]$ ). 
In [2] we have mentioned that $\left|\operatorname{Aut}\left(\mathbb{Z} / 2 \mathbb{Z}\left[\Delta_{2}\right]\right)\right|=6$. It follows that $\mid \operatorname{Aut}(\mathbb{Z} /$ $\left.2 \mathbb{Z}\left[\Delta_{1}\right]\right)|\cdot| \operatorname{Aut}\left(\mathbb{Z} / 2 \mathbb{Z}\left[\Delta_{2}\right]\right) \mid=6$. It turns out that in this case the formula $\operatorname{Aut}(W)=\operatorname{Aut}\left(\mathbb{Z} / 2 \mathbb{Z}\left[\Delta_{1}\right]\right) \times \operatorname{Aut}\left(\mathbb{Z} / 2 \mathbb{Z}\left[\Delta_{2}\right]\right)$ is not true. In fact we cannot describe the group $\operatorname{Aut}(W)$. However, we have shown that $|\operatorname{Aut}(W)|=24$ with the help of complex computer program (see algorithm of our program in [7]).

Unfortunately we have no tool for describing the group of strong automorphisms of Witt rings which are direct products of any group Witt rings.

\section{References}

[1] Marshall M., Abstract Witt Rings, volume 57 of Queen's Papers in Pure and Applied Math. Queen's University, Ontario 1980.

[2] Stępień M.R., Stępień L., On strong automorphisms of products of Witt rings (I), Journal of Applied Mathematics and Computational Mechanics 2013, 12, 3, 123-133.

[3] Stępień M.R., Automorphisms of Witt rings and quaternionic structures, Scientific Research of the Institute of Mathematics and Computer Science 2011, 1(10), 231-237.

[4] Stępień M., Automorphisms of Witt rings, unpublished PhD thesis, University of Silesia, Katowice 2010 (in Polish).

[5] Stępień M., Automorphisms of products of Witt rings of local type, Acta Mathematica et Informatica Universitatis Ostraviensis 2002, 10, 125-131.

[6] Stępień M., Automorphisms of Witt rings of elementary type, [in:] Mathematica. Proceedings of the XI ${ }^{\text {th }}$ Slovak-Polish-Czech Mathematical School, Pedagogical Faculty Catholic University in Ružomberok 2004, June $2^{\text {nd }}-5^{\text {th }}, 62-67$.

[7] Stępień L., Stępień M.R., Automatic search of automorphisms of Witt rings, Scientific Issues. Mathematics, XVI, Jan Długosz University, Częstochowa 2011, 141-146. 